\title{
Drug utilization study in intensive cardiac care unit of tertiary care hospital
}

\author{
Madhuri D Kulkarni ${ }^{1}$, Siddharth S Athawale ${ }^{2 *}$ \\ ${ }^{1}$ Professor and HOD, ${ }^{2}$ Assistant Professor, Dept. of Pharmacology, Government Medical College and Hospital, Aurangabad, Maharashtra, \\ India
}

*Corresponding Author: Siddharth S Athawale

Email: siddharthathawale20@gmail.com

\begin{abstract}
Introduction: Patients admitted in Intensive Cardiac Care unit (ICCU) are critically ill cardiac patients. Cardiovascular diseases (CVDs) are the main cause of mortality globally and are the leading cause of death in India also. The Intensive Cardiac Care unit is routinely equipped with a system that permits continuous monitoring of the cardiac rhythm of each patient and hemodynamic monitoring in selected patients. The various drugs used in ICCU and there clinical outcome was investigated in this study.

Materials and Methods: Patients admitted between January 2017 - June 2018 in Medicine Intensive cardiac care unit were included in this study.

Results: Total of 359 prescriptions was analyzed from the Intensive cardiac care unit. Out of 359 patients, $228(63.51 \%)$ were male, while $131(36.49 \%)$ were female. The mean age of the patients admitted in ICCU was $55.68+13.54$ years. The most common type of patients admitted in ICCU have the diagnosis of Acute myocardial infarction (77.16\%), followed by Ischemic heart disease (8.36\%), Cardiogenic shock (4.18\%), Left Bundle Branch Block (3.62\%), Rheumatic heart disease (2.79\%) and Supraventricular tachycardia (2.23\%). The most common drugs prescribed in ICCU were Aspirin, Clopidegrol and Atorvastatin. Other drugs most commonly used are Metoprolol (95.82\%), Ramipril (92.48\%), Isosorbitedinitrate (86.07\%), Ranitidine (83.29\%).

Conclusion: In conclusion, after analyzing the prescriptions we found that the drugs used in ICCU like anti-platelets (clopidogrel and aspirin), anti-coagulants (LMWH), statins (atorvastatin) and nitrates (Isosorbidedinitrate) were in large quantities. This finding correlates with the standard recommended guidelines for cardiovascular emergencies. The present study provides valuable insight about the overall pattern of drugs used in intensive cardiac care unit.
\end{abstract}

Keywords: Drug utilization study, Intensive cardiac care unit, Anti-platelets.

\section{Introduction}

The World Health organization (WHO) in 1997 defined drug utilization as "the marketing, distribution, prescription and use of drugs in a society, with special emphasis on the resulting medical, social and economic consequences". ${ }^{1}$ Patients admitted in ICCU are critically ill cardiac patients. ${ }^{2}$ Cardiovascular diseases (CVDs) are also the main cause of mortality globally and are the leading cause of death in India. ${ }^{3,4}$ In India, CVDs are the largest cause of mortality, accounting for around one-fourth of all deaths. ${ }^{5}$ The Intensive Cardiac Care unit (ICCU) is well-equipped unit with continuous monitoring of the cardiac rhythm of each patient and hemodynamic monitoring in selected patients. The duration of stay in the intensive cardiac care unit is decided by the ongoing need for intensive care. If symptoms are controlled with oral and parenteral therapy and when the patient is stable, then they are transferred out of the intensive care unit. Also, patients who have a confirmed STElevation Myocardial Infarction (STEMI) but who are considered to be at low risk (no prior infarction and no persistent chest discomfort, Congestive heart failure, hypotension, or cardiac arrhythmias) may be safely transferred out of the cardiac care unit within 24 hour.

Therefore, by studying the prescription pattern of the ICCU patients it will give an idea about the use of drug pattern in this institution and will be helpful for creating favorable conditions for wide scale improvements in therapeutic practices in our setup. Therefore, the main objective of this study was to assess the utilization of drugs, whichwere given in patients admitted in the Intensive cardiac care unit in a government tertiary care hospital.

\section{Materials and Methods}

A prospective study was conducted from January 2017 to June 2018 in the Intensive cardiac care unit of tertiary care hospital.

\section{Inclusion criteria}

1. Patients of either sex of more than 12 years.

2. Patients admitted in Intensive cardiac care unit.

3. Patients or their relatives who are willing to give informed consent.

\section{Exclusion criteria}

1. Patient or their relatives not willing to give informed consent.

2. Patients who stayed for less than 24 hours.

3. Incomplete data entry case records were excluded from the study.

Approval from ethics committee was taken. Data collected was of age, duration of stay in ICCU, final diagnosis, drugs prescribed and clinical outcome of patients from case papers. The data obtained was then analyzed in Microsoft excel. Qualitative data are expressed in frequency \& percentage while quantitative data are expressed with mean \pm standard deviation (SD). 


\section{Observations and Results}

The prospective observational study was carried out at tertiary care teaching hospital in Intensive cardiac care unit. Total prescription of 359 patients was analyzed from Intensive cardiac care unit.Detailed information about baseline demographic characteristics and treatment received by the patient was studied. Observations of the study are presented in the form of different tables and figures.

Out of 359 patients, $228(63.51 \%)$ were male, while $131(36.49 \%)$ were female. The mean age of the patients admitted in ICCU was $55.68+13.54$ years. The average duration of stay in ICCU was 2.57 days. Out of 359 patients admitted in ICCU, $286(79.67 \%)$ patients were treated and transferred to other wards or discharged, $67(18.66 \%)$ patients were expired, while $6(1.67 \%)$ patients were discharged against medical advice. (Table 1)

The most common type of patients admitted in ICCU have the diagnosis of Acute myocardial infarction (77.16\%), followed by Ischemic heart disease $(8.36 \%)$, Cardiogenic shock (4.18\%), Left Bundle Branch Block (3.62\%), Rheumatic heart disease $(2.79 \%)$ and Supraventricular tachycardia (2.23\%). (Table 2)

\section{Drug utilization pattern}

A total of 359 patients from ICCU received 3924 drugs, number of drugs prescribed per patient being $10.93 \pm 3.22$ (mean \pm standard deviation). (Table 5)

The most common drugs prescribed in ICCU were Aspirin, Clopidegrol and Atorvastatin. Other drugs most commonly used are Metoprolol (95.82\%), Ramipril (92.48\%), Isosorbitedinitrate $\quad(86.07 \%), \quad$ Ranitidine $(83.29 \%)$. (Table 3)

The total number of drugs prescribed per prescription in ICCU is most commonly between9-12 were (56.55\%) drugs, between $13-17$ were $29.25 \%$ and between 5-8 were $10.86 \%$. (Table 4)
Using WHO drug prescription indicator, collected data were analyzed and found that average number of drugs prescribed per encounter in ICCU was $10.93 \pm 3.22$.

Percentage of encounters with injection in ICCU was $50.4 \%$.

Percentage of drugs from National list of essential medicine in ICCU was $80.6 \%$.

Percentage of drugs from WHO essential medicine list in ICCU was $64.5 \%$. (Table 5)

Table 1: Demographic details

\begin{tabular}{|l|c|}
\hline Demographic & Frequency $(\%)(\mathbf{n}=359)$ \\
\hline Male & $228(64 \%)$ \\
\hline Female & $131(36 \%)$ \\
\hline Age (Mean+SD) & $55.68+13.54$ years \\
\hline Length of ICU Stay & 2.57 days \\
\hline Outcome \\
\hline Transferred to ward & $286(79.67 \%)$ \\
\hline DAMA & $6(1.67 \%)$ \\
\hline Expired & $67(18.66 \%)$ \\
\hline
\end{tabular}

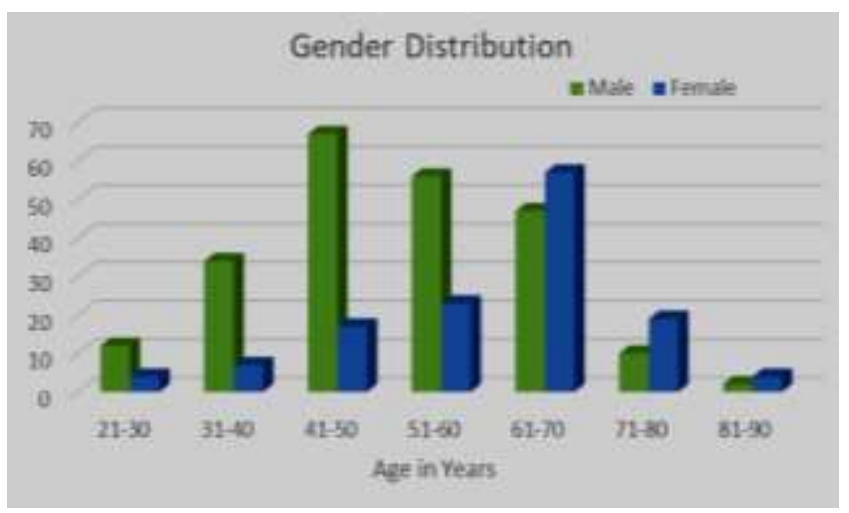

Fig. 1: Gender distribution in ICCU

Table 2: Types of Diagnosis in ICCU

\begin{tabular}{|c|c|c|c|}
\hline S. No. & Diagnosis & No. of Patients & Percentage \\
\hline 1 & Acute myocardial infarction & 277 & $77.16 \%$ \\
\hline 2 & Ischemic heart disease & 30 & $8.36 \%$ \\
\hline 3 & Cardiogenic shock & 15 & $4.18 \%$ \\
\hline 4 & Left Bundle Branch Block & 13 & $3.62 \%$ \\
\hline 5 & Rheumatic heart disease & 10 & $2.79 \%$ \\
\hline 6 & Supraventricular tachycardia & 8 & $2.23 \%$ \\
\hline 7 & Ventricular tachycardia & 4 & $1.11 \%$ \\
\hline 8 & Atrial fibrillation & 2 & $0.56 \%$ \\
\hline & Total & $\mathbf{3 5 9}$ & $\mathbf{1 0 0 \%}$ \\
\hline
\end{tabular}

Table 3: Various drugs used in ICCU

\begin{tabular}{|c|c|c|c|}
\hline Drugs & No. of Prescriptions & Percentage & ATC Code \\
\hline Aspirin & 345 & $96.10 \%$ & B01AC06 \\
\hline Atorvastatin & 345 & $96.10 \%$ & C10AA05 \\
\hline Clopidogrel & 345 & $96.10 \%$ & B01AC04 \\
\hline Metoprolol & 344 & $95.82 \%$ & C07AB02 \\
\hline Ramipril & 332 & $92.48 \%$ & C09AA05 \\
\hline Isosorbitedinitrate & 309 & $86.07 \%$ & C01DA08 \\
\hline
\end{tabular}




\begin{tabular}{|c|c|c|c|}
\hline Ranitidine & 299 & $83.29 \%$ & A02BA02 \\
\hline Dopamine & 163 & $45.40 \%$ & C01CA04 \\
\hline Morphine & 161 & $44.85 \%$ & N02AA01 \\
\hline Streptokinase & 147 & $40.95 \%$ & B01AD01 \\
\hline Hydrocortisone & 146 & $40.67 \%$ & H02AB09 \\
\hline Pentazocine & 130 & $36.21 \%$ & N02AD01 \\
\hline Nicorandil & 113 & $31.48 \%$ & C01DX16 \\
\hline Theophylline & 111 & $30.92 \%$ & R03DA04 \\
\hline N-Acetylcysteine & 104 & $28.97 \%$ & R05CB01 \\
\hline Ondansetron & 100 & $27.86 \%$ & A04AA01 \\
\hline Dalteparin & 94 & $26.18 \%$ & B01AB04 \\
\hline Methyl prednisolone & 64 & $17.83 \%$ & H02AB04 \\
\hline Ethamsylate & 50 & $13.93 \%$ & B02BX01 \\
\hline Lactulose & 50 & $13.93 \%$ & A06AD11 \\
\hline Tranexemic acid & 50 & $13.93 \%$ & B02AA02 \\
\hline Noradrenaline & 27 & $7.52 \%$ & C01CA03 \\
\hline Furosemide & 25 & $6.96 \%$ & C03CA01 \\
\hline Verapamil & 18 & $5.01 \%$ & C08DA01 \\
\hline Glyceryl trinitrate & 15 & $4.18 \%$ & C01DA02 \\
\hline Cefotaxim & 10 & $2.79 \%$ & J01DD01 \\
\hline Mannitol & 10 & $2.79 \%$ & B05BC01 \\
\hline Dobutamine & 7 & $1.95 \%$ & C01CA07 \\
\hline Amiodarone & 6 & $1.67 \%$ & C01BD01 \\
\hline Digoxin & 2 & $0.56 \%$ & C01AA05 \\
\hline Diltiazem & 2 & $0.56 \%$ & C08DB01 \\
\hline
\end{tabular}

Table 4: Total no. of drugs per prescription ICCU

\begin{tabular}{|l|c|c|}
\hline No. of Drugs & No. of Prescription & Percentage \\
\hline $9-12$ & 203 & $56.55 \%$ \\
\hline $13-17$ & 105 & $29.25 \%$ \\
\hline $5-8$ & 39 & $10.86 \%$ \\
\hline $1-4$ & 12 & $3.34 \%$ \\
\hline Total & $\mathbf{3 5 9}$ & $\mathbf{1 0 0 . 0 0 \%}$ \\
\hline
\end{tabular}

Table 5: WHO core indicators

\begin{tabular}{|l|l|c|}
\hline S.No & Core indicators & Percentage \\
\hline & & ICCU \\
\hline 1 & Total number of drugs prescribed & 3924 \\
\hline 3 & Average no. of drugs per prescription & $\mathbf{1 0 . 9 \%}(3924 / 359)$ \\
\hline 3 & Total encounters having injectable formulation & $\mathbf{5 0 . 4 \%}(1981 / 3924)$ \\
\hline 5 & Drugs mentioned in NLEM 2015 & $\mathbf{8 0 . 6 \%}$ \\
\hline
\end{tabular}

\section{Discussion}

Emergency Medicine it is a field of practice based on the knowledge and skills required for the prevention, diagnosis and management of acute and urgent aspects of illness affecting patients. A patient admitted to an ICCU presents a challenge to the attending doctors. Prompt and early detection of cardiovascular emergencies and immediate initiation of therapy are therefore necessary for reduction in mortality and morbidity due to cardiovascular emergencies.

Total 359 patient's case data was studied from ICCU. The data which was collected included demographic information of the patient (age and sex), diagnosis of the patient, treatment given, duration of stay in ICCU and there clinical outcome.

In ICCU the number of males admitted was $63.51 \%$ while that of female were $36.49 \%$. (Table 1 ) This shows that more male patients were affected as compare to female patients. These results are in adherence with standard literature, which state that estrogen increases high-density lipoproteins (HDL) and lowers low-density lipoproteins (LDL), whereas the androgens have the opposite effect. Estrogen in addition has a direct vasodilator effect. Thus endogenous estrogens exert a cardio protective influence. ${ }^{6}$ Similar results were shown in Indian studies done by Nagabushan $\mathrm{H}$ et al. having $64.7 \%$ males and $35.3 \%$ 
females, Patil et al. having $66.9 \%$ males and $33.1 \%$ females, Jyothi et al. having $68 \%$ males and $32 \%$ females respectively. ${ }^{7-9}$ Also in the study of 'acute myocardial infarction' conducted by M. Martinez et al. $80 \%$ were males and $20 \%$ females. $^{10}$ In another study of myocardial infarction conducted by Ian A. Scott et al. $66.3 \%$ of patients were males and $33.7 \%$ females respectively. ${ }^{11}$

The mean age of the patients admitted in ICCU was $55.68+13.54$ years. The mean age of male patients was $52.20+16.66$ years while that of female patients was 59.64 $+18.17 \mathrm{yrs}$. (Table 1 ) In the age group $31-50$ years, the number of female patients was found significantly less as compare to the number of female patients in the age group 51-70 years. The reason for increased incidence of cardiovascular emergencies in female could be the loss of cardio-protective effect of estrogen after menopause. In addition, there was no significant difference between number of male and female patients in the age group 61-70 years. (Fig. 1)

The mean duration of stay in ICCU was $2.57+0.91$ days (Table 1) which is comparable to studies done by Patel et al. $3.07 \pm 1.39$ days and Jyothi et al. 3 days respectively. ${ }^{2,9}$

In ICCU, out of 359 patients, $77.16 \%$ had Myocardial infarction, which was by far the most common cardiovascular anomaly resulting in ICCU admission, followed by Ischemic heart disease $(8.36 \%)$ (Table 2) which was similar to studies done by Pendhari S R et al. and Jyothi et al. respectively. ${ }^{4,9}$ Myocardial Infarction included both ST elevated myocardial infarction (STEMI) in which majority had anterior wall MI followed by inferior wall MI \& Non ST elevated myocardial infarction (NSTEMI). Hypertension and diabetes were the two most common co-morbid conditions found in most of the patients, which were in accordance with study conducted by Jyothi et al., Dawalji et al. and Christian et al. respectively. ${ }^{9}, 12$

Among the drugs used in ICCU, in our study we noticed that utilization rate of drugs like antiplatelets (Aspirin, Clopidogrel), Anticoagulant (LMWH), Statin (Atorvastatin), Anti-hypertensives (Metoprolol, Ramipril), Nitrates (Isosorbidedinitrate) was in large quantities. (Table 3) This finding correlates with the standard guidelines mentioned for use of drug in cardiovascular emergencies. These results were found to be similar to various studies conducted by Nagabushan et al., Ian A. Scott et al., F Venturini et al. ${ }^{7,11,13}$

Further, in this study it was seen that utilization rate of ACE inhibitors and beta blockers was much more than calcium channel blockers. This correlates with the present trends of increased use of ACE inhibitors in cardiovascular diseases. According to study done by Friedman B.M, recent data from the mega trial support the early use of ACE inhibitors after acute MI. In this mega trial, the use of ACE inhibitors was associated with substantial reduction in mortality in MI patients. ${ }^{14}$ Furthermore, the ACE inhibitors had a beneficial effect against fibrosis and cardiac remodeling. ${ }^{15} \mathrm{M}$. Martinez et al during their study between 1989-1994 on trends of prescribing patterns in patients with
Myocardial infarction noticed that the proportion of patients prescribed an ACE inhibitor increased from 14\% in 1989 to $23 \%$ in 1994 and a continued decrease in the use of calcium channel blockers was noted from $36 \%$ in 1989 to $17 \%$ in $1994 .{ }^{10}$ Streptokinase was the most commonly used fibrinolytic though newer plasminogen activators with less side effects available, as streptokinase is less expensive and effective.

In this study, morphine sulfate $(44.85 \%)$ was the most commonly utilized analgesic followed by Pentazocine $(36.21 \%)$. (Table 3) This finding was similar to previous studies carried out by John et al., Biswal et al. ${ }^{16,17}$ Morphine is the principal sedative agent preferred in ICCU due to its potency, improved patient ventilator synchrony, and availability of effective antagonist. Pentazocine causes tachycardia and rise in BP at higher doses due to sympathetic stimulation. This may increase cardiac work, so it should be avoided in coronary ischaemia and myocardial infarction. The beta-agonists such as Dopamine or Dobutamineare used to improve hemodynamics, in acutely decompensated chronic heart failure patients dopamine or dobutamine or noradrenaline were used in combination with intravenous fluids for the patients of septic and cardiogenic shock. They were used to restore cardiac output, tissue perfusion and oxygenation. Inotropes are mainly effective in early stages of shock.

\section{Limitations of the study}

1. In this study, we had not taken the prescription data after the patients were transferred from ICCU to other wards.

2. Defined daily dose was not calculated which would have given a more knowledge on drug utilization.

3. The utilization of intravenous fluids and various drug interaction among the prescribed drugs was not accounted.

\section{Future out looks}

1. Education to the healthcare professional.

The outlook of this study is to conduct interventional studies in order to compare the use and efficacy and cost effectiveness of two drugs within the same class in order to prepare an efficacious hospital formulary plan.

\section{Conclusion}

In conclusion, we noticed that the most common emergencies in ICCU were myocardial infarction and ischemic heart disease. It was also seen that these emergencies were more common in males than in females.

After analyzing the prescriptions we found that in ICCU drugs like antiplatelets (clopidogrel and aspirin), anticoagulants (LMWH), statins (atorvastatin) and nitrates (Isosorbidedinitrate) were used in large quantities. This finding correlates with the standard recommended guidelines for cardiovascular emergencies.The present study provides valuable insight about the overall pattern of drugs used in Intensive cardiac care unit. 


\section{Source of Funding}

None.

\section{Conflict of Interest}

None.

\section{References}

1. World Health Organization international working group for drug statistics methodology. Introduction to drug utilization research.

http://apps.who.int/medicinedocs/pdf/s4876e/s4876e.pdf. 2003;1-48

2. Patel BJ, Patel KH, Trivedi HR. Drug Utilization Study In Intensive Coronary Care Unit of A Tertiary Care Teaching Hospital. Natl J Integr Res Med. 2012;3(4):28-33.

3. Thaker V PK. A study of drug utilization pattern in post-acute coronary syndrome (ACS) patients at tertiary care teaching hospital: a prospective unicentric study. Int J Basic Clin Pharmacol. 2017;6(2):308-311.

4. Pendhari SR, Chaudhari DR, Burute SR, Bite BM. A study on the drug utilization trends in the cardiovascular emergencies in a tertiary care hospital. J Clin Diagnostic Res. 2013;7(4):66670.

5. Lakshmi SK, Gowda HN, Sadananda KS. A study of drug utilization trends in acute coronary syndrome in intensive cardiac care unit at a tertiary care hospital, Mysore. Int J Basic Clin Pharmacol. 2017;6(2):344-8.

6. Dunaif A. Womens health. In: Harrison's principles of internal medicine 19th ed. Mc Graw Hill Medical publishing div; 2015:6e-2.

7. Nagabushan H, Roopadevi HS, Prakash GM, Pankaja R. A prospective study of drug utilization pattern in cardiac intensive care unit at a tertiary care teaching hospital. IJBCP Int J Basic Clin Pharmacol. 2015;4(3):579-83.
8. Patil BV, Binjawadgi A, Dass P, MichellGulabani SP. Drug utilisation study in intensive cardiac care unit at a tertiary care hospital. J Evol Med Dent Sci. 2013;2(28):5239-50.

9. Jyothi CH, Vidya HK, Dinakar KR, Shashikala GH. Drug utilization study in intensive cardiac care unit of a tertiary care hospital. Int J Pharma Res Heal Sci. 2015;3(5):824-30.

10. Martinez M, Agustí A, Arnau JM, Vidal X, Laporte JR. Trends of prescribing patterns for the secondary prevention of myocardial infarction over a 13-year period. Eur J Clin Pharmacol. 1998;54:203-8.

11. Scott IANA, Heath K, Harper C, Clough A. An Australian comparison of specialist care of acute myocardial infarction. Int J Qual Heal Care. 2003;15(2):155-61.

12. Dawalji S, Venkateshwarlu K, Thota S, Venisetty PK, Kumar R. Prescribing pattern in coronary artery disease: A prospective study. Int J Pharma Res Rev. 2014;3(3):24-33.

13. Venturini F, Romero M, Tognoni G. Acute myocardial infarction treatments in 58 Italian hospitals. Ann Pharmacother. 1995;29:1100-5.

14. Friedman BM, City K. Early Interventions in the Management of Acute Uncomplicated Myocardial Infarction. West J Med. 1995;162:19-27.

15. Tripathi KD. Cardiovascular Drugs. In: Essentials of Medical Pharmacology. 7th ed. Jaypee Brothers Medical Publishers (P) LTD; 2013;504.

16. John LJ, Devi P, John J, Guido S. Drug utilization study of antimicrobial agents in medical intensive care unit of a tertuart care hospital. Asian J Pharm Clin Res. 2011;4(2):2-5.

17. Biswal S, Mishra P, Malhotra S, Puri GD, Pandhi P. Drug utilization pattern in the intensive care unit of a tertiary care hospital. J Clin Pharmacol. 2006;46(8):945-51.

How to cite this article: Kulkarni MD, Athawale SS. Drug utilization study in intensive cardiac care unit of tertiary care hospital. Indian J Pharm Pharmacol 2020;7(1):34-8. 